\title{
Facilities for Nano Materials Examination at the PULSTAR Reactor
}

\author{
A. I. Hawari, M. Liu, Q. Cai \\ Nuclear Reactor Program, Department of Nuclear Engineering \\ North Carolina State University, Raleigh, NC 27695, USA \\ ayman.hawari@ncsu.edu
}

\begin{abstract}
The PULSTAR is a 1-MWth nuclear research reactor located at North Carolina State University. It is fueled by uranium dioxide assemblies enriched to $4 \%$ or $6 \%$ in U-235 and is currently under licensing for operation at 2-MWth power. The PULSTAR is a center for irradiation testing and pre/post irradiation examination of materials. Among its unique capabilities are positron annihilation spectroscopy (PAS) and neutron powder diffraction (NPD) facilities. The PAS facility provides an intense positron beam reaching $6 \times 10^{8} \mathrm{e}^{+} / \mathrm{s}$, which drives two spectrometers; the $\mathrm{e}^{+}$-PAS and the Ps-PAS, used for studies of defects in thin film materials. A Na-22 bulk PAS system is also operational, which is used for studying millimeter scale materials. All spectrometers are capable of performing Doppler Broadening Spectroscopy (DBS) and Positron Annihilation Lifetime Spectroscopy (PALS). To date, the PAS systems have been used to characterize various materials (unirradiated and irradiated) that include graphite, soft matter, and metal-organic frameworks (MOF). The NPD facility uses a double focusing single crystal silicon rotating monochromator producing neutron beams with different energies. A position sensitive detection bank covers a scattering angle of $5^{\circ}$ $125^{\circ}$. The facility is used in the examination of unirradiated and irradiated materials including graphitic materials, magnetic materials without rare-earth elements, and anode materials used in lithium batteries. The collected diffraction patterns can be processed to produce atomic pair distribution functions. The PAS and NPD facilities are available through user programs including the US DOE's Nuclear Science User Facilities (NSUF) and the US NSF's Research Triangle Nanotechnology Network (RTNN).
\end{abstract}

KEYWORDS: PULSTAR, nuclear reactor, positron annihilation, neutron diffraction, nanotechnology

\section{INTRODUCTION}

The PULSTAR nuclear reactor was established as the fourth in a series of reactors on the campus of North Carolina State University (NCSU). It achieved first criticality and started operations in 1972. The PULSTAR is a light water moderated and cooled open pool in tank reactor [1]. The core is configured in $5 \times 5$ array of assemblies with beryllium reflectors of two sides. Each assembly contains an array of $5 \times 5$ (25) fuel pins that contain sintered uranium dioxide pellets enriched to either $4 \%$ or $6 \%$ in U-235 (per assembly) and clad with zircaloy 2 . The total U-235 in the core is nearly $13 \mathrm{~kg}$ and the volume fraction of light water to fuel in the core is nearly unity. The PULSTAR was originally designed as a pulsing reactor with the maximum pulse power reaching $2200 \mathrm{MWth}$. However, since 1983 the pulsing operation of the PULSTAR ceased and only steady state operation is permitted by its current license. 
Figure 1 below shows the arrangement of the PULSTAR core including surrounding beam tubes. In addition, calculated spectral and spatial distributions of neutrons in the PULSTAR core are given showing that the thermal neutron flux (energy less than $0.5 \mathrm{eV}$ ) is largest at core's periphery and near the beam tubes' entry planes. Once recognized, this design feature provided further motivation for utilization of the reactor and developing various beamline capabilities. Consequently, over the past 18 years, the PULSTAR was outfitted with several unique facilities including the positron annihilation spectroscopy (PAS) facility on beam tube \#6, and the neutron powder diffractometer (NPD) on beam tube \#4 [2]. Figure 2 shows the current PULSTAR bay area and a corresponding schematic layout of the various facilities.
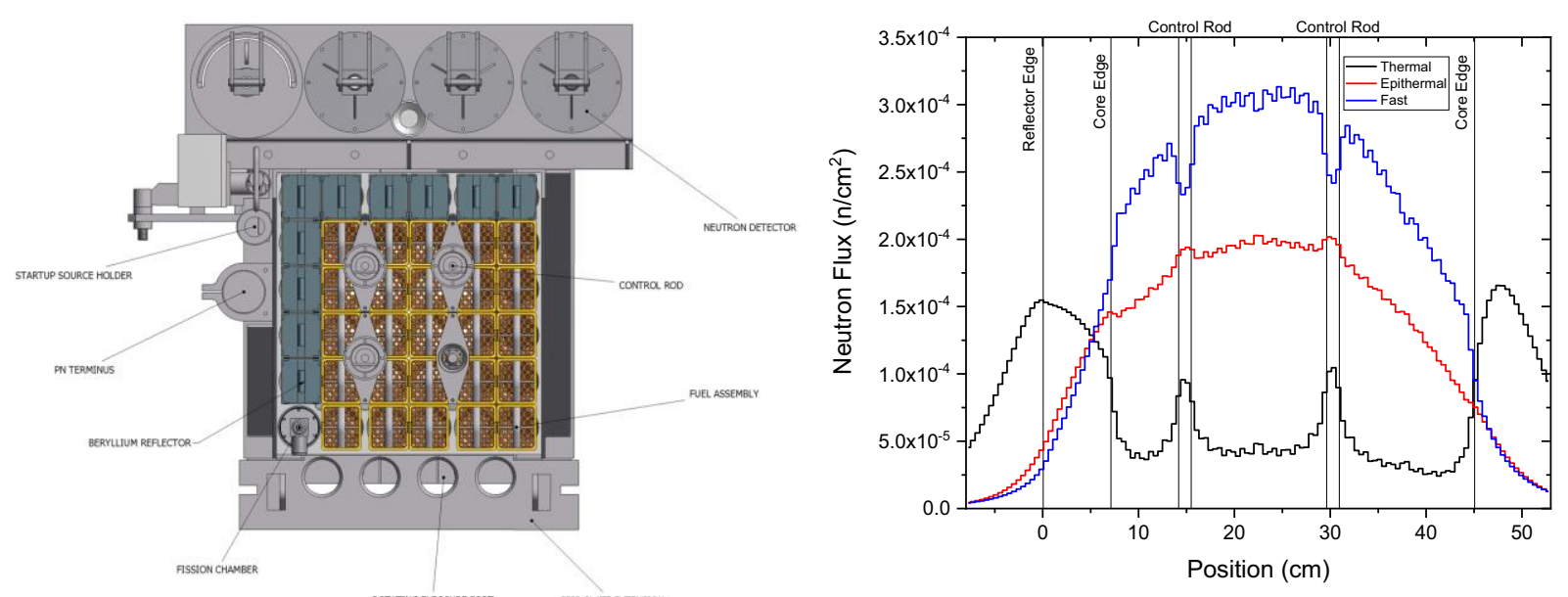

Fig. 1. A layout of the PULSTAR's core including the fuel assemblies and the beryllium reflectors appearing on the top and left side of the core (left). The calculated energy and spatial distribution of neutrons in the PULSTAR core illustrating the large flux of thermal neutrons (energy less than 0.5 $\mathrm{eV}$ ) in the core's periphery (right).
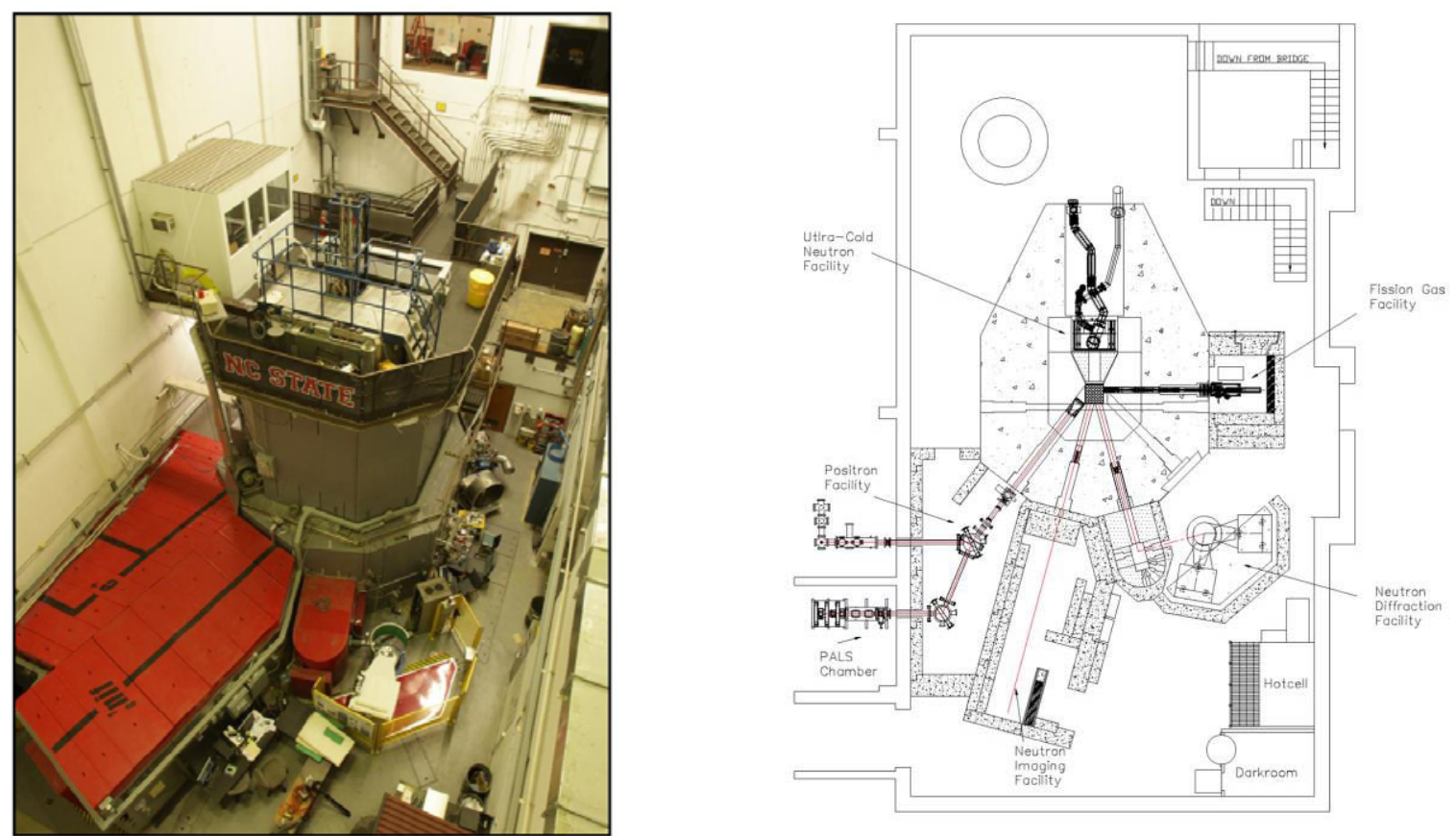

Fig. 2. A view of the PULSTAR reactor's bay floor (left). The corresponding layout of the bay floor showing the various facilities on each beam tube. 


\section{The Positron Annihilation Spectroscopy Facility and Applications}

Positron annihilation spectroscopy (PAS) has been proven to be a powerful and non-destructive tool for nano materials characterization, especially for studying the vacancy and free volume properties at the atomic level of a wide range of materials $[3,4]$. Techniques such as positron annihilation lifetime spectroscopy (PALS) measures the decay lifetime of positrons in the subject materials, which is directly related to the electron density surrounding the annihilating positron and is very sensitive to the concentration and type of vacancy defect and defect clusters. In semiconductors and insulators, positrons can also form positronium, a meta-stable bound state of a positron and an electron. Therefore, PALS can also refer to positronium annihilation lifetime spectroscopy. Another positron technique, Doppler Broadening Spectroscopy (DBS), examines the energy spread of the $511 \mathrm{keV}$ back-to-back annihilation gamma-ray originating from the moving electron when the annihilation occurs and provides complementary information of the vacancy type and density in the energy domain.

\subsection{The PULSTAR Intense Positron Beam}

In the past decade, an intense positron beam facility with two associated spectrometers was established at the PULSTAR nuclear reactor [5,6]. In addition, a digital bulk positron spectrometer has been established. A schematic of the intense beam user facility is shown in Fig. 3 below. Positrons are produced through pair production in a Platinum converter, which is irradiated by high energy gamma-rays from the reactor core and from neutron capture in a cadmium end-cap surrounding the source. Positrons emitted from the Platinum with kinetic energies of a few $\mathrm{eV}$ are accelerated and focused electrostatically into a magnetic field for transport out of the bio-shield. This results in a $25 \mathrm{~mm}$ diameter beam with an energy of $1 \mathrm{keV}$ and intensity of $\sim 6 \times 10^{8}$ positrons/second. The primary positron beam is magnetically transported out of the high radiation area through magnetic switches and into adjacent labs that house the two positron annihilation spectrometers (Fig. 3). These spectrometers are set up to conduct positron annihilation lifetime spectroscopy (PALS) and Doppler Broadening Spectroscopy (DBS) of annihilation irradiation. Other positron methodologies could also be implemented per users' request. In addition to the beam-based spectrometers described above, a Na-22 based bulk PAS system is also currently operational (Fig. 4). The system has complete digital signal processing and data acquisition. The source-sample package can be placed on a heating-cooling stage inside a vacuum chamber. The temperature of thermal treatment to the sample ranges from approximately $80 \mathrm{~K}$ to $600 \mathrm{~K}$. The sample stage is positioned in between two photomultiplier tube (PMT) assemblies in combination with two cylindrical plastic scintillators of 1 inch $\times 1$ inch in size. The pulses from the PMTs are digitized and processed in real time to extract timing information, which results in approximately $170 \mathrm{ps}$ timing resolution. Meanwhile, an analog system runs in parallel with the digital system for higher count rate experiments with less requirement on timing resolution. The beam and bulk systems can be used for the study of vacancy, vacancy cluster, or void concentration and size in various types of materials of micrometer to millimeter level thickness.

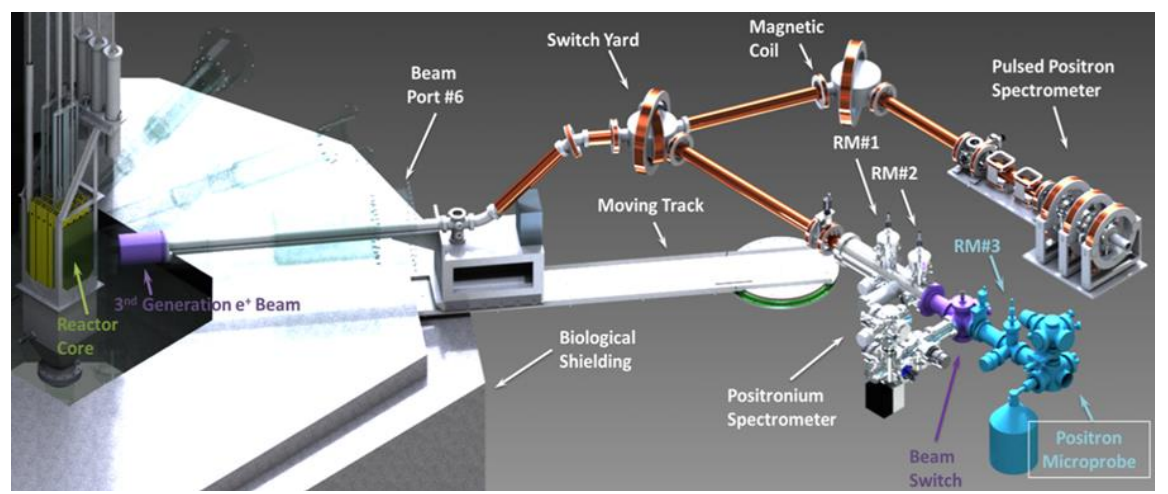

Fig. 3. Schematic of the intense positron beam user facility at the PULSTAR reactor. 

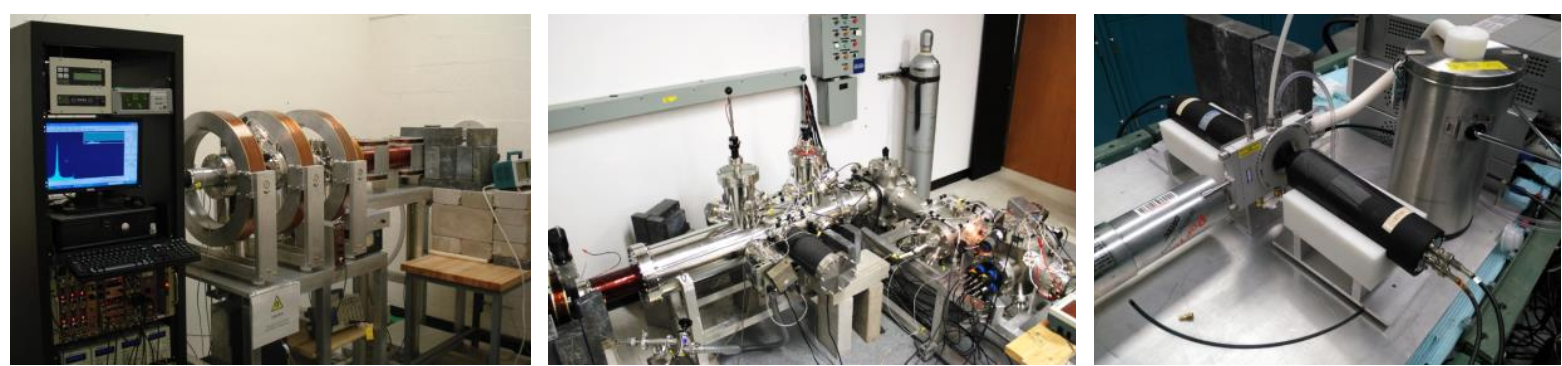

Fig. 4. the e ${ }^{+}$-PAS spectrometer (left), Ps-PAS spectrometer (center), and bulk spectrometer (right).

\subsection{PAS Applications at the PULSTAR Reactor}

The PAS facility at the PULSTAR reactor has been used in various studies to investigate the nanostructure of materials by implementing the PALS and DBS techniques discussed above. An example is nuclear graphite, which is utilized as a neutron moderator/reflector as well as a structural component in various concepts of advanced reactors, wherein the graphite is exposed to an intense flux of neutrons that introduces displacement of lattice atoms and creates vacancy and interstitial Frenkel pairs. The migration and agglomeration of these point defects leads to effects that can alter both the structural and neutronic behavior of the graphite. In a previous work [7], radiation damage of nuclear graphite induced by neutrons was examined by PALS and specific vacancy types have been identified that were associated with the irradiation process and the atomic mobility (Fig. 5). This result has also been correlated to the microstructural changes observed by neutron diffraction patterns [8], and used to support computer simulation of nuclear graphite [9].
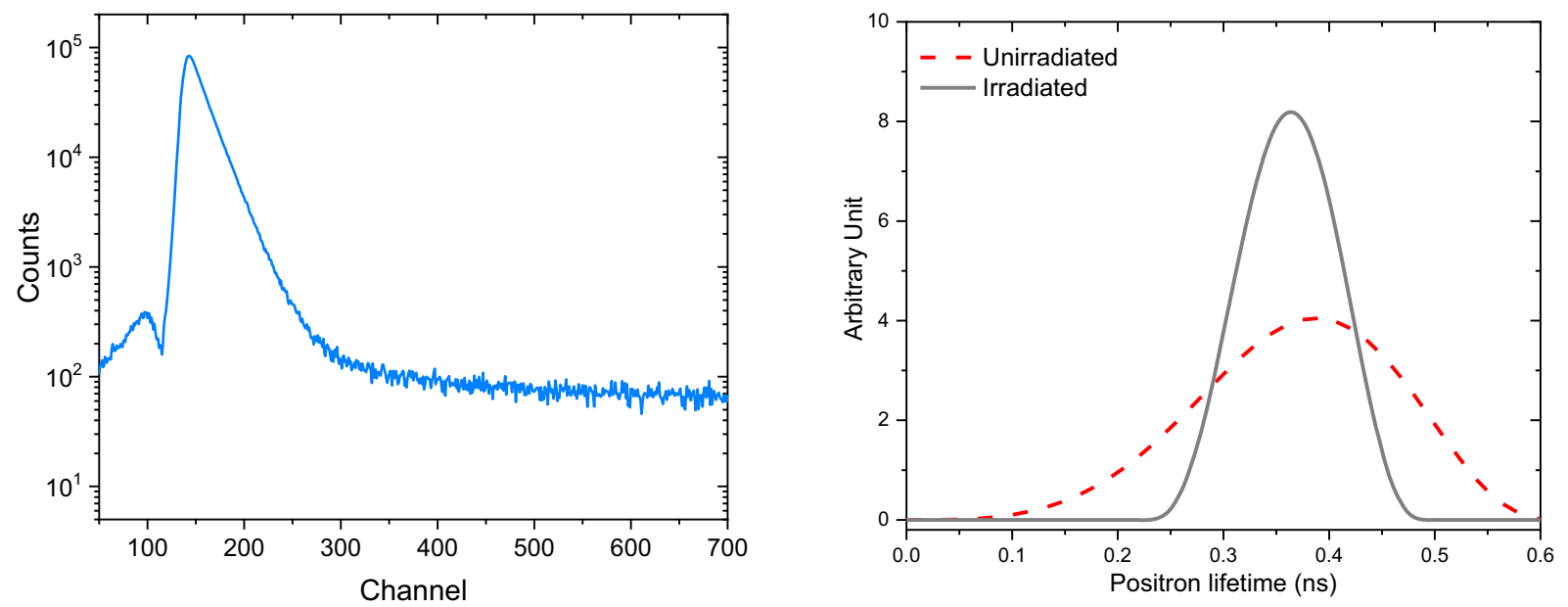

Fig. 5. PALS spectrum (left), and lifetime distribution (right) in nuclear graphite samples [7].

A valuable feature of PAS is that it can be utilized to monitor the changes of free volumes in-situ in samples under their operational conditions. For example, the swelling behavior of Cellulose nanofibrils in the presence of water [10]. In this study, severity of the swelling behavior of these films was found to be related to film drying temperatures during fabrication. Meanwhile, it is also crucial to clarify and separate the effect of positronium (Ps) annihilation in the free volumes and Ps bubble formation in water. A direct relationship has been established between the microscopic structural change due to water molecules and macroscopic sorption behavior. This sorption behavior is not limited to water swelling but also various gas adsorption processes, for instance, in metal-organic frameworks (MOFs) [11]. As a whole category of materials, MOFs have shown unprecedented surface areas and tunable pore sizes that could be used for a 
variety of purposes including adsorption, separation, sensing, and filtering. In these systems, the Ps lifetime can be directly related to the nanopore size and its change during gas adsorption, which could hugely improve our understanding of the crystal quality and its adsorption dynamics.

\section{The Neutron Powder Diffractometer Facility and Applications}

Neutron powder diffraction (NPD) is an analytical technique that aims at studying the structural characteristics of atomic and molecular systems. Neutron diffraction has various advantages over similar techniques (e.g., X-ray diffraction) including penetrability and the ability to interact with light nuclei such as hydrogen. In NPD the data is analyzed using the Rietveld refinement technique. In this case, a powder diffraction pattern of a polycrystalline material is thought of as a collection of individual reflection profiles, each of which has a profile function, a peak height, a peak position, a breadth, and an integrated area which is proportional to the Bragg intensity. This intensity is proportional to the square of the absolute value of the structure factor. By adjusting the parameters of an assumed structure model, the Rietveld method establishes a best fit between the observed intensity and calculated intensity. Therefore, detailed crystal structure information is derived from powder diffraction using the entire measured diffraction pattern without allocating in advance observed intensities to particular Bragg peaks.

\subsection{The PULSTAR NPD Facility}

The Neutron Powder Diffractometer (NPD) facility is located at beam tube \#4 of the PULSTAR reactor [12]. Two 3-inch thickness single crystal sapphire filters are employed as fast neutron and high energy gamma-ray filters. A double focusing single crystal silicon monochrometer, which can focus twodimensional neutrons to the sample position, is employed. Monochromatic neutron beams with different neutron energy can be produced by the monochrometer. A bank of 15 position sensitive detectors is employed, spanning a range of $20^{\circ}$, which can be moved from $15^{\circ}$ to $115^{\circ}$ to cover the entire scattering angle of $5^{\circ}-125^{\circ}$. An Oscillating Radical Collimator (ORC), located at the front of the detector shielding, reduces the background by stopping neutrons scattered from locations other than the sample position. A fission chamber located at the beam, before the sample, is employed as a neutron flux monitor. The detectors collect data at each bank position for a fixed monitor count to ensure that the incoming neutrons at the sample position is similar for each position. The collected data from the 6 positions is combined into a full scattering angle range data. Recently, the pulse processing electronics and the detector bank motors and their drivers were upgraded. Figure 6 shows the NPD facility. The characteristics of the NPD are shown in Table 1. Typically, neutrons with a wavelength of $1.478 \AA$ are used in the experiments, which are reflected by the (511) planes of silicon.

\subsection{NPD Applications at the PULSTAR Reactor}

The NPD facility was used in the examination of various materials to understand fundamental structure and to explore the impact on performance. The studies also included materials that have been exposed to neutron irradiation to explore radiation damage effects. An example is nuclear graphite [8], where the diffraction measurements showed that, upon irradiation, the displaced carbon atoms favor going to the large inter planar space to increase the average interlayer " $\mathrm{d}$ " spacing and its fluctuation. At the same time, the existence of the interstitial atoms and loops will force their neighbor layers to shift randomly, and the probability of random shifting increased. Furthemore, the corresponding vacancy defects in the crystal lattice distort the lattice in the plane locally. This distortion causes contraction along the a axis and the entire lateral dimension. The vacancy defects in the crystal lattice may join together to form vacancy loops that collapse the planar structure of graphite. Figure 7 below shows the measured diffraction patterns and the related pair distribution functions that are derived as the Fourier transforms of the patterns. 

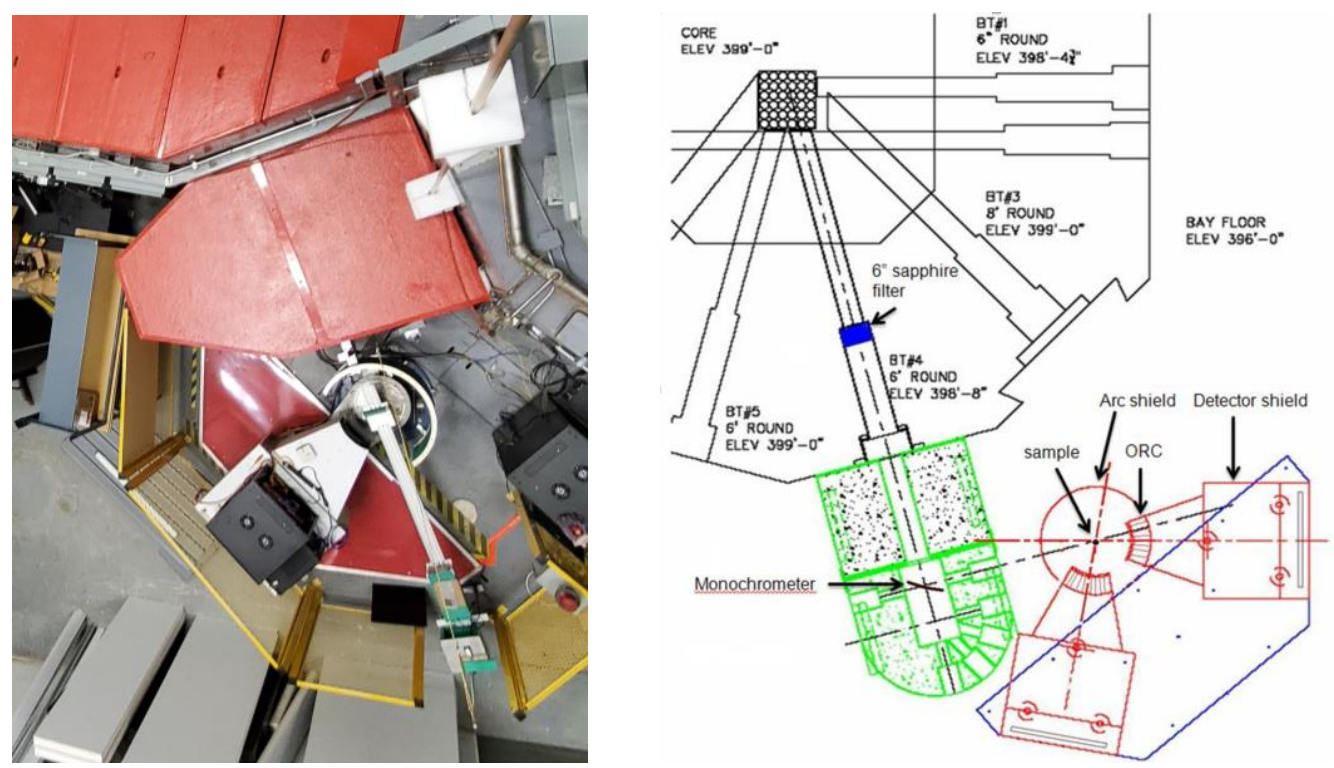

Fig. 6. A view of the NPD facility and its corresponding schematic.

Table 1. Characteristics of NPD at the PULSTAR Reactor

\begin{tabular}{|l|l|}
\hline Beam Spectrum & Thermal \\
\hline Monochromator & Bent silicon single crystal monochromator \\
\hline Take off angle & $2 \Theta_{\mathrm{m}}=90^{\circ}$ \\
\hline Wavelength & $1.075 \AA, 1.171 \AA, 1.478 \AA, 1.567 \AA, 1.762 \AA, 2.316 \AA$ \\
\hline Scattering angles & $5^{\circ} \leq 2 \Theta \leq 125^{\circ}$ \\
\hline Detector bank: & $15 \mathrm{He}-3$ detectors \\
\hline Beam size at sample position: & $7 \mathrm{~cm} \times 1 \mathrm{~cm}$ \\
\hline Minimum resolution $(\Delta \mathrm{d} / \mathrm{d}):$ & $3 \times 10^{-3}$ \\
\hline
\end{tabular}

Another study examined the structure of $\mathrm{MnAl}$, which represents a ferromagnetic material. The $\tau$-phase of $\mathrm{MnAl}$ has received considerable attention because of its high magnetic moments, high magnetic crystalline anisotropy, and high Curie temperature [13]. In this case, neutron diffraction measurements were performed on the Mn-Al alloy with atomic ratio 54:46 to study its crystal structure and magnetic ordering. The diffraction pattern is shown in Figure 8, the refinement results showed that the crystal structure of $\mathrm{Mn}_{54} \mathrm{Al}_{46}$ is tetragonal (space group $\mathrm{P} 4 / \mathrm{mmm}$ ) with lattice parameters $\mathrm{a}=2.7778 \AA$, and $\mathrm{c}=3.5773 \AA$. The 1a site is occupied by Mn atom mostly, and the $1 \mathrm{~d}$ site is occupied by the Al atom mostly. The Mn atoms at the same sites have ferromagnetic ordering, but the $\mathrm{Mn}$ atoms at different sites are coupled antiferromagnetically. The magnetic moment of $\mathrm{Mn}$ atoms is about $2.03 \mu_{\mathrm{B}}$, and its direction is along the $\mathrm{c}$ axis. The unbalance in occupation of $\mathrm{Mn}$ atoms at $1 \mathrm{a}$ and $1 \mathrm{~d}$ sites is the origin of the permanent magnetic property of this alloy.

A timely example is lithium-ion batteries (LIBs), which are widely used in portable electronic products, plug-in hybrid vehicles, and even electric vehicles (EVs). However, the low specific capacity of the commercial LIB cathodes limits the further use in EVs. Therefore, it is highly desired to find new cathodes with high capacity. The Li-rich Mn-based layered oxides are considered as candidates to replace the current commercial LIB cathodes due to their large discharge capacity, low cost, and good thermal stability [14]. However, the disadvantages of voltage decay, large irreversible capacity loss in the first cycle, and the poor rate capability hinder their mass application. Extensive studies have been done to overcome these problems through surface modifications and metal ion substitution. In this work, Ti doped Li-rich layered oxides $\mathrm{Li}_{1.2} \mathrm{Mn}_{0.6-\mathrm{x}} \mathrm{Ni}_{0.2} \mathrm{Ti}_{\mathrm{x}} \mathrm{O}_{2}(\mathrm{x}=0.0,0.006)$ cathode material were studied using neutron diffraction measurement. 
The measured diffraction pattern is given below (Fig. 8), and by using Rietveld refinement, shows that the samples have hexagonal structure with R-3m space group. Ti doping ( $3 \mathrm{~b}$ sites) can increase the lattice parameters. The occupancies of $\mathrm{Li}$ and $\mathrm{Ni}$ at $3 \mathrm{a}$ and $3 \mathrm{~b}$ sites are mostly unimpacted due to the Ti doping. In addition, the diffraction patterns are characteristic of almost a single phase structure.
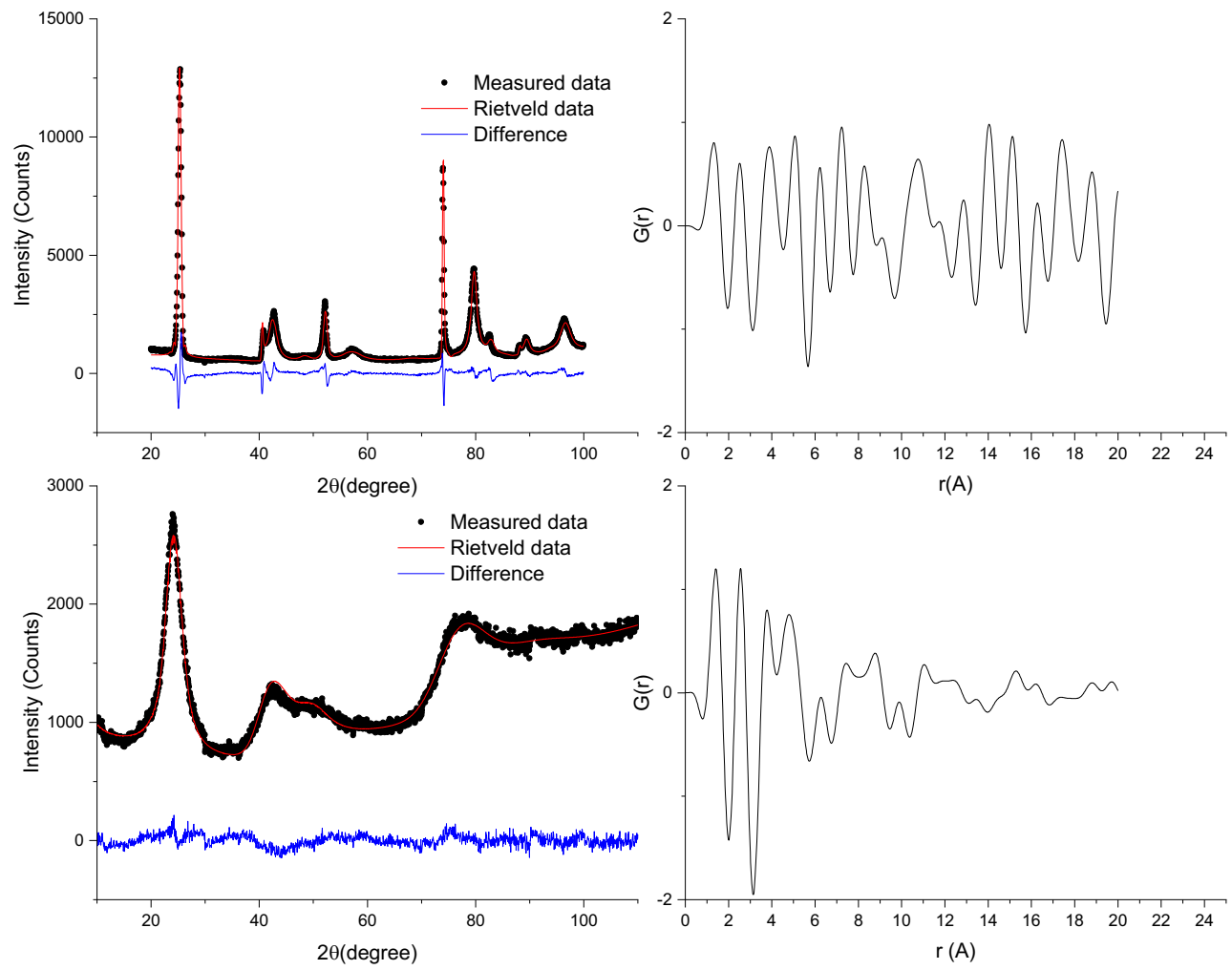

Fig. 7. Neutron powder diffraction patterns and PDF of nuclear graphite samples. The top plots represent unirradiated samples. The bottom plots are for irradiated samples showing the loss of the planar graphite structure.
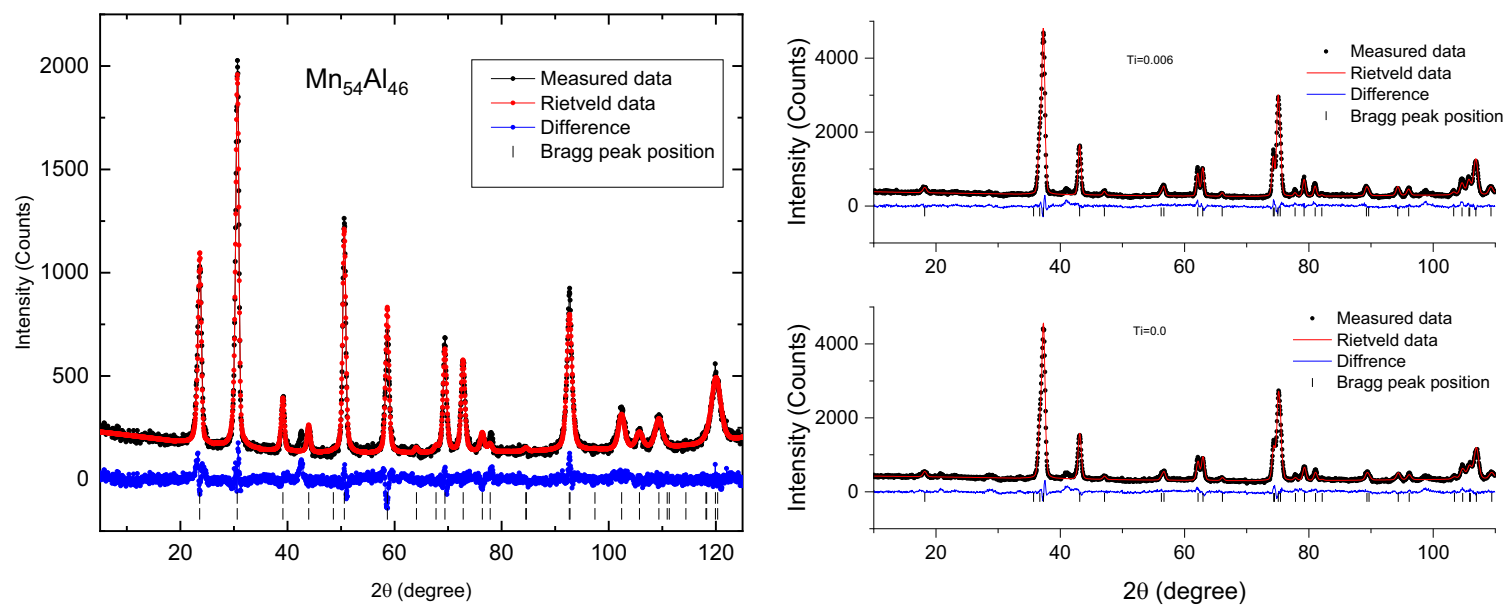

Fig.8. Neutron powder diffraction pattern of $\mathrm{Mn}_{54} \mathrm{Al}_{46}$ (left), and of the $\mathrm{Ti}$ doped and undoped Lirich layered oxides $\mathrm{Li}_{1.2} \mathrm{Mn}_{0.6-\mathrm{x}} \mathrm{Ni}_{0.2} \mathrm{Ti}_{x} \mathrm{O}_{2}(\mathrm{x}=\mathbf{0 . 0}, 0.006)$ cathode material (right). 


\section{CONCLUSIONS}

Two unique facilities for PAS and NPD characterization of the nanostructure of materials have been developed and operated at the NCSU PULSTAR reactor. The facilities take advantage of the intimate coupling between the PUSTAR core and its surrounding beam tubes to achieve optimum performance. The PAS facility includes an intense beam $\left(6 \times 10^{8}\right.$ positrons/second $)$ and bulk spectrometers that are ideal for performing both PALS and DBS analyses. The NPD facility is capable of a resolution of $3 \times 10^{-3} \Delta \mathrm{d} / \mathrm{d}$. Both facilities are used for characterizing various materials including graphite, MOFs, steel, semiconductors, various types of soft matter, ferromagnetic materials, and lithium-ion battery materials. By having such facilities, the PULSTAR continues its mission as a center of pre/post irradiation examination of materials.

\section{ACKNOWLEDGMENTS}

The PAS and NPD facilities and their utilization are funded by the US Department of Energy through its INIE, NEUP and NSUF programs and by the US National Science Foundation through its MRI and RTNN programs.

\section{REFERENCES}

1. J. MacPhee, J. F. Wett, "PULSTAR-The AMF Pulsing Reactor," Research Reactor Journal, 4(12), pp. 12-19 (1963).

2. A. I. Hawari, "Multidisciplinary Engagement at Research Reactors: The NCSU PULSTAR," Proceedings of IGORR 2017, Sydney, Australia (2017).

3. Y. C. Jean, P. E. Mallon, D. M. Schrader, Principles and applications of positron and positronium chemistry, World Scientific, River Edge, NJ, USA (2003).

4. D. W. Gidley, H-G Peng, R. S. Vallery, "Positron Annihilation as A Method to Characterize Porous Materials", Annual Review of Materials Research, 36, pp. 49-79 (2006).

5. A. I. Hawari, et al., "Operation and testing of the PULSTAR reactor intense slow positron beam and PALS spectrometers," Journal of Physics: Conference Series, 262 (2011).

6. M. Liu, et al., "The Intense Slow Positron Beam Facility at the PULSTAR Reactor and Applications on Nano-materials Study," AIP Conf. Proc., 1525 (2013).

7. M. Liu, A. I. Hawari, "Positron Characterization of Neutron Irradiated Reactor-Grade Graphite," Transactions of the American Nuclear Society, 110, (2014).

8. Q. Cai, A. I. Hawari, "Neutron Powder Diffraction Study of Reactor Grade Graphite," Embedded Topical Meeting on Nuclear Fuels and Structural Materials for the Next Generation Nuclear Reactors, Chicago, IL, USA (2012).

9. A. I. Hawari, V. H. Gillette, "Inelastic Thermal Neutron Scattering Cross Sections for Reactor-Grade Graphite," Nuclear Data Sheets, 118, pp. 176-178 (2014).

10. J. Ø. Torstensen., et al. "Swelling and free-volume characteristics of TEMPO-oxidized cellulose nanofibril films." Biomacromolecules, 19(3), pp. 1016-1025 (2018).

11. S. Nandi, et al. "A single-ligand ultra-microporous MOF for precombustion $\mathrm{CO}_{2}$ capture and hydrogen purification," Science advances, 1(11), pp. 1-9 (2015).

12. D. D. DiJulio, A. I. Hawari, "Characteristics and Performance of the Neutron Powder Diffractometer at the NC State University PULSTAR Reactor," PHYSOR-2008: International Conference on the Physics of Reactors, Nuclear Power: A Sustainable Resource, Interlaken, Switzerland (2008).

13. J. M. D. Coey, New permanent magnets; manganese compounds, Journal of Physics: Condensed Matter, 26(064211), pp. 1-6 (2014).

14. A. Manthiram, et al., "Nickel-Rich and Lithium-Rich Layered Oxide Cathodes: Progress and Perspectives," Advanced Energy Materials, 6(1501010), pp. 1-23 (2016). 